persistence or remission. The prognosis for ADHD seems to be bleaker than previously perceived. By using stimulant medication, special education, and counselling during early grade school years, the improvements in attentiveness and behavior are expected to result in better study habits and academic achievement. To assure continued success in high school and also, in college, ADHD appears to require persistent medical attention. Treatment should also be directed more aggressively toward lessening the influence of environmental and familial adverse factors, which appear to be important in persistence of ADHD. Parents are often oblivious or in denial of their role in the etiology of a child's attention deficits and behavior problems. Family counselling, often neglected as part of the multimodal therapy for ADHD, should receive greater emphasis.

Psychiatric and developmental disorders in families of children with ADHD were studied in the Department of Pediatrics, Wyler and La Rabida Children's Hospitals, University of Chicago. (Roizen NJ et al. Arch Pediatr Adolesc Med Feb 1996;150:203-208). Children with ADHD were significantly more likely than control children (with Down syndrome) to have a parent affected by alcoholism, other drug abuse, depression, delinquency, learning disabilities, and ADHD. Anticipatory guidance and psychosocial intervention were recommended for families affected. The Editor, Dr DeAngelis, notes that children with a family history of psychiatric disorders should be screened for ADHD.

\title{
CARBAMAZEPINE: A THERAPY FOR ADHD
}

The efficacy of carbamazepine (CBZ) in treatment of ADHD has been determined by meta-analysis of 10 reports from the international literature reviewed at Columbia University, St Luke's-Roosevelt Hospital Center, and New York University Medical Center. In 7 open studies involving a total of 189 patients with features of motor overactivity, impulsivity, and distractibility, $70 \%$ showed a marked improvement in target symptoms following treatment with CBZ for periods varying from 1 week to 8 years. Outcome was significantly correlated with duration of treatment; the longer the treatment the better the outcome. In 3 placebo-controlled, double-blind studies, $71 \%$ of 53 patients treated with CBZ were benefited whereas only $26 \%$ of 52 receiving placebo showed similar improvement in attentiveness and behavior. The difference was significant $(\mathrm{p}=.018)$. The most frequent side effects were sedation and skin rash occurring in $7.5 \%$ and $5.7 \%$ of CBZ-treated patients, respectively. (Silva RR et al. Carbamazepine use in children and adolescents with features of attention-deficit hyperactivity disorder: a meta-analysis. I Am Acad Child Adolesc Psychiatry March 1996;35:352-358). (Reprints: Dr Silva, St Luke's/Roosevelt Hospital Center, Division of Child and Adolescent Psychiatry, 411 W114th Street, Suite 3A, New York, NY 10025).

COMMENT. The authors concluded that carbamazepine may be an effective alternate treatment for ADHD. A response rate of $70 \%$ in both open and controlled studies is about the equivalent effectiveness of stimulant medication.

From a neurologist's perspective, the obvious questions would relate to the incidence of epilepsy and epileptiform EEG's in these patients selected for treatment with an anticonvulsant medication. Unfortunately, these data were not discussed and were tabulated for the entire sample and not the subsample with ADHD. My own meta-analysis of these data show that abnormal EEGs occurred in $69 \%$ of 57 patients in the controlled studies and in $82 \%$ of 50 
patients in the one open study providing EEG data. Seizures were mentioned in 4 of the studies, affecting 13 plus patients, but the total number of patients affected was not given. The frequency of abnormal EEGs in these patients is considerably higher than that usually reported for ADHD. It seems that CBZ might be indicated for the treatment of ADHD symptoms in some patients with abnormal EEGs and/or a history of seizures. On a negative note, see Progress in Pediatric Neurology II, 1994, pp188-190, for references to cognitive impairment and impulsivity caused by CBZ treatment of epilepsy.

The cognitive effects of carbamazepine, phenobarbital, and valproate were compared in 73 children with newly diagnosed epilepsy studied at the National Cheng Kung University; Chi Nei Hospital; and Tainan Municipal Hospital, Tainan, Taiwan, ROC. (Chen Y-J, Kang W-M, Chin-Min So W, Epilepsia 1996;37:81-86). Only children treated with phenobarbital showed increased P300 latencies on auditory event-related potentials, which was inversely related to IQ scores after treatment for 6 to 12 months. WISC-R IQs and Bender-Gestalt scores were not significantly different in any of the groups before or after treatment. P300 latency was a more sensitive indicator of AED effects on cognitive function than the WISC-R and Bender-Gestalt.

Behavioral side effects of Gabapentin are reported in 7 children with base-line ADHD and developmental delays who were followed in the epilepsy program and in the Department of Child Psychiatry, Emory University, Atlanta, GA. (Lee DO et al. Epilepsia 1996;37:87-90). Tantrums, aggression, hyperactivity, and defiance were the most troublesome symptoms. The majority (64\%) of intensified behaviors were similar to baseline ADHD symptoms; $21 \%$ were ODD and $8 \%$ were CD symptoms. New behaviors, not exhibited before gabapentin therapy, were ODD or CD. Behavioral side effects resolved after decrease or withdrawal of gabapentin.

\section{BRAIN INJURY IN INFANCY AND LEARNING DISABILITIES}

Dyscalculia and dyslexia in a 17-year-old boy after right hemisphere injury in infancy is reported from the Division of Neurosurgery and Department of Pediatrics, University of Maryland, Baltimore; and Cognitive Neuroscience Section, National Institutes of Health, Bethesda, MD. Social behavior was normal, but math and spelling abilities were impaired and his attention span was short. A functional MRI showed predominantly left hemisphere activation involving frontal and posterior parietal regions while the patient performed calculations. In normal subjects this test produced bilateral activation of the supramarginal gyrus. These MRI findings were consistent with early interhemisperic transfer of right parietal visuospatial skills to the left parietal region. Dyscalculia and dyslexia with normal IQ suggest an acquired left parietal dysfunction caused by competition for left hemisphere representation between verbal and visuospatial functions. (Levin HS et al. Dyscalculia and dyslexia after right hemisphere injury in infancy. Arch Neurol Jan 1996;53:88-96). (Reprints: Dr Grafman, Cognitive Neuroscience Section, NIH/NINDS/MNB, Bldg 10, Room 5S 209, 10 Center Dr, MSC 1440, Bethesda, MD 20892).

COMMENT. The authors conclude that interhemispheric reorganization of function and language may be bidirectional and not only a left hemisphere feature of language development. The MRI showed an intact left hemisphere following the injury. Visuospatial functions normally subserved by the right parietal area were probably transferred to the left parietal region, causing a crowding effect and disproportionate impairment of reading and math skills 